\title{
Perception of a Stepping-across Affordance when Approaching an Obstacle
}

\author{
Cornus Sabine \\ Faculté des Sciences du Sport, Université de Strasbourg \\ cornus@unistra.fr
}

\begin{abstract}
Affordances are behavior opportunities that delineate a phase transition beyond which a change in behavior requiring muscular reorganization. In the stepping across task, this muscular reorganization enables subjects to put the tip of the shoe as close as possible to the obstacle. But subjects modify step lengths when approaching an obstacle to secure these adequate foot placements. What is the relationship between the perception of the phase transition and the modification of step lengths?

This experiment consisted of two different tasks. The first involved subjects physically stepping across an obstacle, the second involved subjects judging the step-acrossability of an obstacle when moving towards the given obstacle. The results showed that subjects seemed to perceive the step-acrossability of the obstacle before the initiation of regulation.
\end{abstract}

\section{Introduction}

The perception of affordances allows the subject to perceive their behavior afforded by the task, the environment and the situation [1]. We know that the subject's capability in perceiving an affordance is dependent on the fact that they are capable of perceiving the pertinent environmental properties that are scaled according to their own abilities. This suggests that the subject perceives the critical point, exploiting it to regulate his/her behavior, thus yielding the appropriate shift such as stepping across to jumping [2]. This critical point delineates an action boundary, i.e., a phase transition beyond which a change in behavior requiring muscular reorganization and different control laws must take place.

Nevertheless, the type of the obstacle forces subjects to place their foot as close as possible to the obstacle boundary [3]. And subjects must regulate the approach phase to reorganize her/his behavior. Cornus et al. [4] have manipulated the obstacle width in the stepping across an obstacle task. Subjects had to step across a $2 \mathrm{~mm}$-high obstacle, $1 \mathrm{~m}$ in length and of variable width (i.e., $1.03 \mathrm{~m}$, Exp. 1; $0.05 \mathrm{~m}, 0.19$ $\mathrm{m}, 0.38 \mathrm{~m}, 0.57 \mathrm{~m}$, Exp. 2). The results showed that the task constraints play an important role: they define at a given step the initiation of regulation. Subjects initiated their regulation more or less late as a function of spatial constraints (i.e., obstacle width). And the high level of spatial constraint (i.e., a large obstacle width) required the subject to distribute adjustments over a large number of steps [4]. They were able to accurately perceive the properties of obstacles and changed their locomotor strategies to provide a safety margin when going over obstacles of different characteristics.

Then, the aim of this study was to identify the distance necessary for perceiving the stepacrossability of an obstacle. In addition, we have to analyze the relationship between this distance and the subject's behavior when approaching an obstacle.

\section{Method \\ Subjects}

Nineteen subjects $(M=27$ years, $S D=6.42)$ volunteered to take part in this experiment. Their mean anthropometric characteristics were as follows: height $1.79 \mathrm{~m}(\mathrm{SD}=0.06)$, leg length .93 $\mathrm{m}(\mathrm{SD}=0.03)$ (e.g., distance from the lateral malleolus to the anterior-posterior iliac spine).

All subjects had normal or corrected vision. Each subject read and signed a written informed consent document outlining the procedures of the experiment. The study was conducted according to the declaration of Helsinki.

\section{Apparatus}

The experiment took place in a gymnasium where subjects were asked to walk on a run-up track $(25 \times 2.30 \mathrm{~m})$. A locometer was used to record the longitudinal displacements of both feet and the distance in which subjects judged to perceive the step-acrossability of the obstacle. A 2mm-high obstacle was designed, $1 \mathrm{~m}$ in width and of variable depth. In the stepping across task, the obstacle depth ranged between $.60 \mathrm{~m}$ and $1.40 \mathrm{~m}$. 
Nevertheless in the judging task, the obstacle depth depended on the subject's actual critical stepping across size $(\mathrm{Sa})$.

\section{Task and procedure}

Subjects performed two different tasks: an obstacle stepping across task and an obstacle step- acrossability judgment task.

In the stepping across task, subjects had to walk naturally between two marks $10 \mathrm{~m}$ apart and step across the obstacle located along the way, $6 \mathrm{~m}$ from the starting point. If the stepping across was too difficult the subject could jump the obstacle. The obstacle depth ranged between $.60 \mathrm{~m}$ and $1.20 \mathrm{~m}$ for subjects whose height was under $1.75 \mathrm{~m}$, and between $.80 \mathrm{~m}$ and $1.40 \mathrm{~m}$ for subjects whose height was above $1.75 \mathrm{~m}$. The obstacle depth was increased or decreased in .05 meter steps with the structure and width of the obstacle remaining constant. Four series, two ascending and two descending, were presented in alternating order. As stipulated in the method of limits, obstacle depths were no longer presented when new behavior was maintained for two consecutive trials $[2,5]$.

In the judging task, the positioning of the obstacle was located at $14 \mathrm{~m}$ from the starting point. Subjects walked towards the obstacle, and when they decided, they stopped to answer the following question: "Would you step across this obstacle". Subjects, with their closed eyes, had to judge the step-acrossability of the obstacle by answering "yes or no". They had three seconds to answer the question. To determine the obstacle depth, we measured the actual critical stepping across size (Sa) of each subject on which basis different obstacle depths were calculated. The depth of obstacles corresponded to $\mathrm{Sa}$ plus or minus 5 or $10 \mathrm{~cm}$ : Sa, Sa-5, Sa+5, Sa-10, Sa+10. The obstacle depths were presented in a counterbalanced order. Two trials per obstacle were performed.

\section{Data analysis procedures}

The analysis was carried out in three phases: the actual critical stepping across size, the free distance, the error percentage and the pattern of the standard deviation of step lengths.

To obtain a measurement of the actual critical stepping across size, the percentage of stepacrossability for each obstacle depth $(\mathrm{n}=13)$, each series $(n=4)$ and each subject $(n=14)$ was analyzed using a logistic function, expressed by the following equation $[2,6]$ : $\%$ of step- acrossability $=100 /\left(1+\mathrm{e}^{-\mathrm{k}(\mathrm{c}-\mathrm{x})}\right)($ equation 1$)$. In equation $1,100 \%$ was the maximum percentage of step-acrossability (the subject always stepped across the obstacles), where " $\mathrm{x}$ " is the obstacle depth in meters. "c" is the $50 \%$ point, that is to say, the obstacle depth at which the subject changed his/her behavior from stepping across to jumping. In other words, this point is the actual critical stepping across size, the boundary of the action. And " $\mathrm{k}$ " is the slope approaching that point.

The free distance (Df) corresponded to the distance chosen by subjects to judge the stepacrossability of the obstacle. $\mathrm{Df}=14 \mathrm{~m}$ (i.e., the distance between the starting point and the obstacle) - D (i.e., the distance calculated by the locometer).

In the judging task, the verbal response obtained for a given obstacle depth (e.g., considered stepable) was compared to the actual critical stepping across size to calculate the percentage of error. $100 \%$ was defined as the percentage of trials judged by subjects to be stepacrossable for an obstacle depth larger than the actual critical stepping across size.

An analysis of variance (ANOVA) was computed on the mean free distances and the mean error percentages. Post-hoc comparisons (i.e., Newman-Keuls test) were used when necessary to examine the nature of the effects. The significance level was set at $\mathrm{p}=.05$.

The standard deviation of step lengths during the approach phase was carried out for each participant $[4,7]$.

\section{Results \\ Actual critical stepping across size}

The mean actual critical stepping across size was equal to $0.90 \mathrm{~m}(\mathrm{SD}=0.06)$.

\section{Free distance}

The analysis of variance revealed a significant effect of the Obstacle factor $(\mathrm{F}(4,56)=8, \mathrm{p}<.05)$ for the mean free distance. A post-hoc comparison (i.e., Newman-Keuls test) showed that the distance chosen by subjects for Sa-10 was significantly different from those chosen in the other three obstacles: $\mathrm{Sa}, \mathrm{Sa}+5, \mathrm{Sa}+10$ (Fig. 1). In addition, the distance chosen by subjects for Sa-5 was significantly different from those chosen in the other two obstacles: $\mathrm{Sa}, \mathrm{Sa}+5$ (Fig. 1). The distances obtained for the smallest obstacles (i.e., Sa-5 and Sa-10) were larger than those obtained in the other three obstacles (i.e., 
$\mathrm{Sa}, \mathrm{Sa}+5$ and $\mathrm{Sa}+10: 6.57 \mathrm{~m}(\mathrm{SD}=3.36), 7.07 \mathrm{~m}$ $(\mathrm{SD}=3.35)$ for $\mathrm{Sa}-5$ and $\mathrm{Sa}-10$ respectively. The distance obtained for $\mathrm{Sa}$ was equal to $5.67 \mathrm{~m}$ $(\mathrm{SD}=3.13)$.

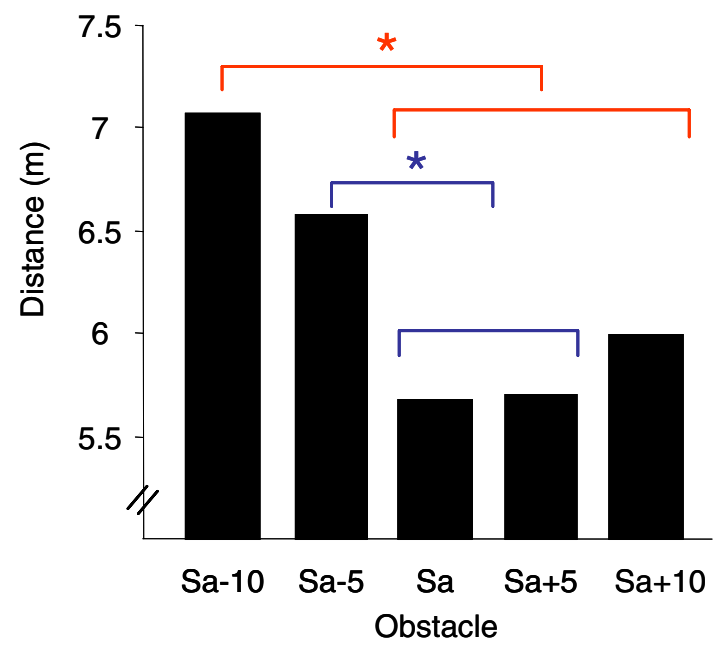

Fig 1. Mean free distances chosen by subjects as a function of the obstacle. The obstacle depth corresponded to the actual critical stepping across size (Sa) plus or minus 5 , or $10 \mathrm{~cm}$.

\section{Error percentage}

The analysis of variance revealed a significant effect of the Obstacle factor $(\mathrm{F}(4,56)=4.71$, $\mathrm{p}<$ $.05)$ for the mean error percentage. A post-hoc comparison (i.e., Newman-Keuls test) showed that the error percentage for $\mathrm{Sa}+5$ was significantly different from those obtained for Sa-10 and Sa-5: 48.33\%, 1.67\%, 15\% for $\mathrm{Sa}+5$, Sa-10, Sa-5 respectively (Fig. 2). Subjects were less accurate for $\mathrm{Sa}+5$.

\section{Pattern of the standard deviation of step lengths}

The mean standard deviation of step lengths was small at the beginning of the approach phase $(\mathrm{SD}=0.02)$ and increased progressively (Fig. 3). It broke over the final step $(\mathrm{S}-1)(\mathrm{SD}=0.14)$.

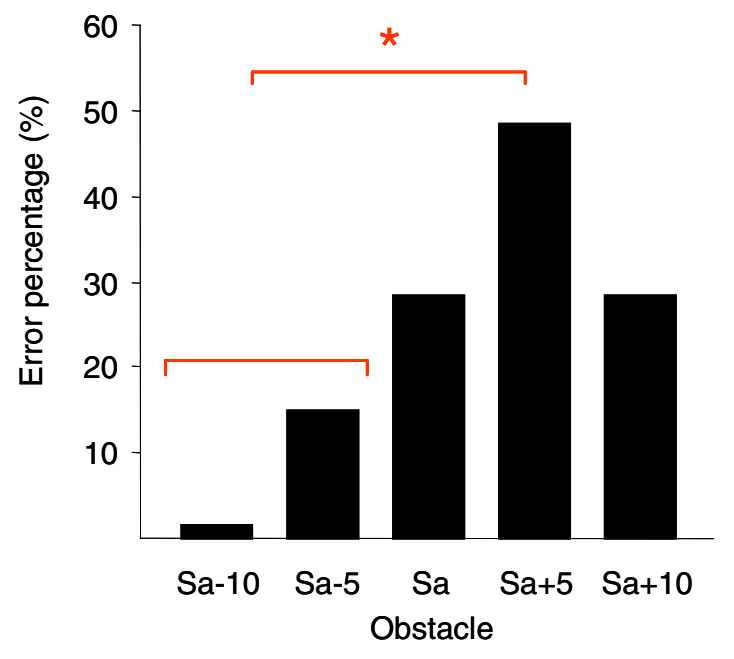

Fig 2. Mean error percentages as a function of the obstacle. The obstacle depth corresponded to the actual critical stepping across size (Sa) plus or minus 5 , or $10 \mathrm{~cm}$.

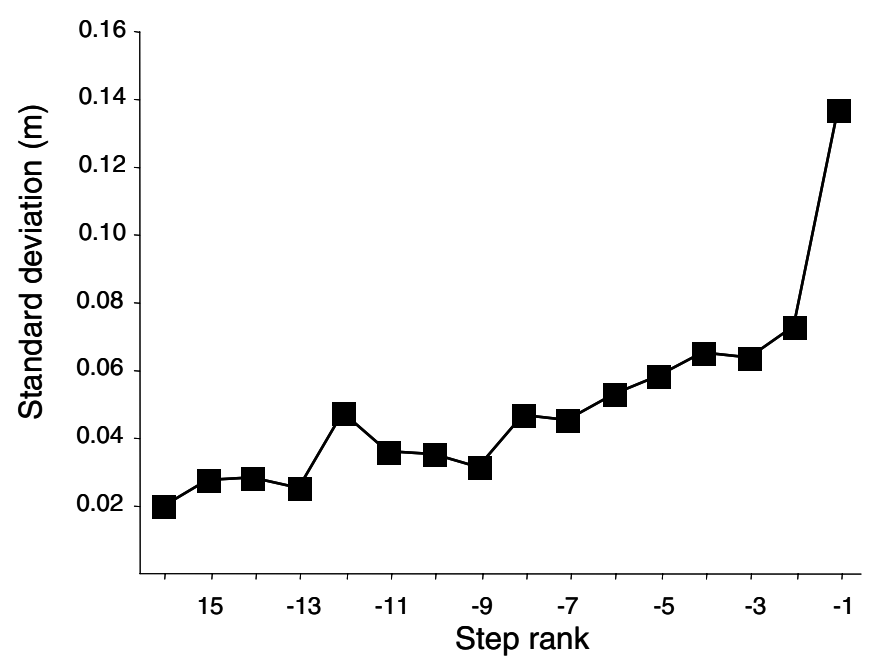

Fig 3. The mean pattern of the SD of step lengths for the last steps where -1 was the last step length before the step-acrossability judgment.

\section{Discussion}

The results showed that the distance at which subjects judged the step-acrossability of the obstacle was not a fixed distance. The obstacle depth influenced the subjects' choice. When the obstacle depth was equal to the actual critical stepping across size $(\mathrm{Sa}=0.90 \mathrm{~m})$, subjects stopped at $5.7 \mathrm{~m}$ before the obstacle. But for Sa10, the distance was equal $7.07 \mathrm{~m}$. The free 
distance was greater when subjects were walking towards the smaller obstacle. Cornus et al. [4] underlined that subjects were able to accurately perceive the properties of obstacles and changed their locomotor strategies to provide a safety margin when going over obstacles of different characteristics. If subjects had to step across a small obstacle, they usually initiated the regulation later: the last or last-but-one step before the obstacle (with a mean obstacle depth, $\mathrm{M}=1.03 \mathrm{~m}$ and a mean step length, $\mathrm{M}=0.78 \mathrm{~m}$ ). Given Bardy and Laurent [8], the braking distance was greater when subjects were walking towards the smaller target $(1.77 \mathrm{~m})$ than the larger target $(1.70 \mathrm{~m})$. Bradshaw and Sparrow [9] in the stepping over a rod task $(0.44 \mathrm{~m}$ in height $)$ found that visual control began on average 4.97 $\mathrm{m}$ from the target. Nevertheless in our study, the distance to judge the obstacle properties was greater than that to initiate the regulation. The difference could be due to subjects' difficulty imagining an action that they do not actually carry out.

Subjects are capable of perceiving their own action boundary, i.e., the obstacle size beyond which they must change from stepping across to jumping. However, the error percentage obtained for $\mathrm{Sa}+5$ was greater than those obtained for the other obstacles. This obstacle depth was close to the actual critical stepping across size. We know that the actual critical stepping across size delineates a phase transition beyond which a change in behavior requiring muscular reorganization. Then, the lack of accuracy could not enable subjects to modify the step lengths.

Given Pailhous et al. [10], the subject maintained consistent step patterns through the small standard deviations of their step lengths (about $0.04 \mathrm{~m}$ on average). But according to Lee et al. [7] in the long jumping task and Cornus et al. [4] in the stepping across task, the standard deviation of step lengths increased during the last steps. This increase of the SD of step lengths underlined the modifications of step lengths to achieve an optimal foot positioning when aiming at the target [7]. In our study, even if subjects did not step across the obstacle, they modified their step lengths. Subject who estimates is less accurate than the subject who engages in action [2]. The fact that the judging action was achieved outside the context in which the actorenvironment system was evolving could account for such a result.

Our study suggests that the perception of a stepping-across affordance and the motor reorganization to place the foot as close as possible to the obstacle depend on a strict coupling between perception and movement.

\section{References}

[1] T.A. Stoffregen. Affordances as properties of the animal-environment system. Ecological Psychology, Taylor \& Francis Group, 15, 115134, 2003.

[2] S. Cornus, G. Montagne, M. Laurent. Perception of a stepping-across affordance. Ecological Psychology, Taylor \& Francis Group, 11, 249267, 1999.

[3] A.E. Patla. Understanding the roles of vision in the control of human locomotion. Gait \& Posture, Elsevier, 5, 54-69, 1997.

[4] S. Cornus, M. Laurent, S. Laborie. Perceptionmovement coupling in the regulation of step lengths when approaching an obstacle. Ecological Psychology, Taylor \& Francis Group, 21, 334367, 2009.

[5] L.S. Mark, D. Vogele. A biodynamic basis for perceived categories of action: A study of sitting and stair climbing. Journal of Motor Behavior, Taylor \& Francis Group, 19, 367-384, 1988

[6] R.J. Bootsma, F.C. Bakker, FEJ. Van Snippenberg, CW. Tdlohreg. The effects of anxiety on perceiving the reachability of passing objects. Ecological Psychology, Taylor \& Francis Group, 4, 1-16, 1992.

[7] D.N. Lee, J.R. Lishman, J.A. Thomson. Regulation of gait in long jumping. Journal of Experimental Psychology: Human Perception and Performance, American Psychological Association, 8, 448-458, 1982.

[8] B.G. Bardy, M. Laurent. Visual cues and attention demand in locomotor positioning. Perceptual and Motor Skills, Ammons Scientific, 72, 915-926, 1991.

[9] E.J. Bradshaw, W.A. Sparrow. Effects of approach velocity and foot-target characteristics on the visual regulation of step length. Human Movement Science, Elsevier, 20, 401-426, 2001.

[10] J. Pailhous, A.M. Ferrandez, M. Flückiger, B. Baumberger. Unintentional modulations of human gait by optical flow. Behavioural Brain Research, Elsevier, 38, 275-281, 1990. 